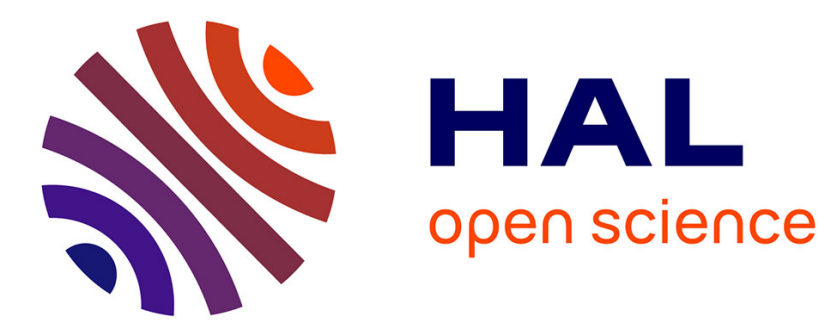

\title{
Le statut de la victime dans la lutte contre la traite des femmes
}

Mathilde Darley

\section{To cite this version:}

Mathilde Darley. Le statut de la victime dans la lutte contre la traite des femmes. Critique Internationale, 2006, 30, pp.103-122. 10.3917/crii.030.0103 . halshs-00648957

\section{HAL Id: halshs-00648957 https://shs.hal.science/halshs-00648957}

Submitted on 30 Aug 2021

HAL is a multi-disciplinary open access archive for the deposit and dissemination of scientific research documents, whether they are published or not. The documents may come from teaching and research institutions in France or abroad, or from public or private research centers.
L'archive ouverte pluridisciplinaire HAL, est destinée au dépôt et à la diffusion de documents scientifiques de niveau recherche, publiés ou non, émanant des établissements d'enseignement et de recherche français ou étrangers, des laboratoires publics ou privés. 


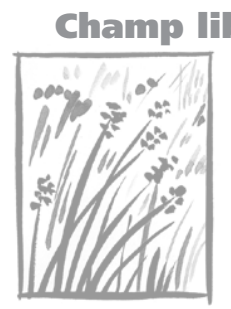

\title{
Le statut de la victime dans la lutte contre la traite des femmes
}

\author{
par Mathilde Darley
}

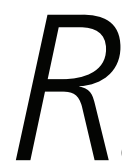

éapparue sur l'agenda politique européen dès $1990^{1}$

et formulée dans les années suivantes en enjeu de politique internationale ${ }^{2}$, la traite des êtres humains figure désormais régulièrement à l'ordre du jour des gouvernements comme des organisations non gouvernementales, nationales ou internationales. Dernier instrument international en date, le Protocole additionnel pour la prévention, la suppression et la répression de la traite des êtres humains, en particulier des femmes et des enfants, ou « Protocole de Palerme $»^{3}$, témoigne de la volonté des différents acteurs en présence de mettre en œuvre une politique anti-traite concertée. Parallèlement, face à la recrudescence du phénomène ${ }^{4}$ et à la dimension intra-européenne que lui

\footnotetext{
1. Le problème de la traite des femmes, popularisé en France avec l'apparition de la notion de « traite des Blanches » à la fin du XIX ${ }^{\mathrm{e}}$ siècle pour rendre compte de l'organisation de migrations massives de femmes européennes vers le Nouveau Monde, constitue tout au long de la première moitié du $\mathrm{XX}^{\mathrm{e}}$ siècle un thème récurrent dans le débat politique international. Pourtant, après l'adoption, en 1949, de la Convention des Nations unies sur la répression de la traite des êtres humains et de l'exploitation de la prostitution d'autrui, la traite des femmes semble provisoirement disparaitre de l'agenda international. Elle n'y réapparaît qu'au cours de la décennie 1990, à la suite notamment de l'ouverture des frontières des États issus du bloc socialiste et du développement des migrations prostitutionnelles de l'Est vers l'Ouest qui l'accompagne. Premier témoin majeur du retour de la traite sur les agendas politiques, un « Séminaire sur la lutte contre la traite des femmes et la prostitution forcée en tant que violations des droits de la personne humaine et atteinte à la dignité humaine » est organisé par le Conseil de l'Europe du 25 au 27 septembre 1991 à Strasbourg.

2. Voir notamment la Conférence mondiale sur les droits de l'homme, Vienne, juin 1993, et la Conférence internationale sur la population et le développement, Le Caire, septembre 1994.

3. Adopté le 15 novembre 2000 par l'Assemblée générale des Nations unies et ouvert à signature en décembre 2000 à Palerme.

4. Conseil de l'Europe, Assemblée parlementaire, Commission sur l'égalité des chances pour les femmes et les hommes, Campagne contre la traite des femmes, Doc. 9190 révisé, 7 septembre 2001.
} 
confère l'élargissement de l'Union à certains pays traditionnellement dits « d'origine », les États membres ont également cherché, avec la Décision cadre relative à la lutte contre la traite des êtres humains adoptée par le Conseil de l'UE en juillet 2002, à conjuguer leurs efforts pour contrer le développement du commerce des êtres humains.

Pourtant, malgré l'actualité brûlante du sujet, son caractère criminel, non quantifiable ${ }^{5}$ et potentiellement dangereux pour l'enquêteur, ainsi que son traitement presque exclusivement « journalistique » semblent encore dissuader les sociologues et les politistes de s'y intéresser. Le présent article s'attachera donc à retracer tout d'abord les conditions d'émergence du problème de la traite sur l'agenda politique international, dans un contexte de multiplication et de transnationalisation des acteurs impliqués, pour étudier ensuite son inscription dans une dialectique discursive opposant deux familles d'acteurs, a priori antagonistes mais qui entretiennent des relations complexes d'interdépendance et partagent finalement une même conception de la victime fondée sur une relative méconnaissance des spécificités de son statut ${ }^{6}$.

\section{Un nouvel enjeu international}

Comme de nombreux objets de politique internationale problématisés au cours du $\mathrm{XX}^{\mathrm{e}}$ siècle en matière de droits universels, la traite des êtres humains (alors appelée «traite des Blanches ») doit aux organisations privées sa première apparition sur l'agenda politique international. En 1899, la National Vigilance Association de Londres, d'obédience protestante, organise le premier congrès international contre la traite ${ }^{7}$, auquel font suite deux conventions internationales relatives à la répression de la traite des Blanches, signées respectivement en 1904 et 1910. Peu après la fin de la première guerre mondiale, la Société des Nations prend l'initiative de deux conférences mondiales qui marquent d'importantes évolutions dans l'acception de la notion de traite : la première, en 1921 , substitue le vocable de « traite des femmes » à

\footnotetext{
5. Il convient de manier avec la plus grande prudence les données quantitatives éventuellement disponibles sur l'ampleur de la traite : le caractère par définition criminel et souterrain des activités liées aux trafics en tout genre rend la collecte de données quasiment impossible et contraint les observateurs à se livrer à diverses estimations, dont l'origine reste obscure et le caractère fluctuant. Reste que ces entreprises de quantification, aussi aléatoires soientelles, n'en décrivent pas moins des réalités semblables et ont de ce fait contribué dans une large mesure à la prise de conscience du phénomène de la traite, et donc à sa constitution en enjeu régional et planétaire.

6. Constat qui ressort notamment des enquêtes de terrain conduites par l'auteur dans les États baltes entre 2002 et 2004. Voir M. Darley, « La lutte contre la traite des femmes en provenance d'Europe de l'Est. Constitution d'un enjeu international de politique publique et observation de la mise en œuvre dans les pays baltes », mémoire de DEA, IEP de Paris, septembre 2004.

7. Alain Corbin, Les filles de noce. Misère sexuelle et prostitution aux $19^{\mathrm{e}}$ et $20^{\mathrm{e}}$ siècles, Paris, Éditions Aubier Montaigne, 1978 , p. 410.
} 
celui de « traite des Blanches », devenu obsolète; la seconde, en 1933, étend la définition de la traite aux femmes majeures consentantes ${ }^{8}$. Au sortir de la seconde guerre mondiale, c'est de nouveau sous l'impulsion d'acteurs privés qu'est adopté le premier instrument international décisif en matière de lutte contre la traite des êtres humains. En 1949, la Convention des Nations unies sur la répression de la traite des êtres humains et de l'exploitation de la prostitution d'autrui réunit l'ensemble des textes internationaux alors disponibles. Elle constituera le texte de référence en la matière jusqu'à l'adoption, en 2000, du Protocole de Palerme 9 . Là encore, les $\mathrm{ONG}$, conviées aux négociations via l'International Human Rights Network, jouent un rôle déterminant dans l'évolution du Protocole, qui devient un instrument de protection de l'ensemble des victimes, ainsi que dans l'inclusion, dans la définition énoncée, de toutes les formes de traite des êtres humains, quand les acceptions précédentes n'avaient retenu que la traite à des fins d'exploitation sexuelle.

Les acteurs privés nationaux peuvent donc être identifiés comme les premiers artisans de la construction des cadres cognitifs ${ }^{10}$ du problème de la traite. Outre la problématisation linguistique du phénomène par le développement de codes de langage appropriés (de la « traite des Blanches », on est passé à la « traite des femmes » puis à la «traite des êtres humains »), ces « entrepreneurs moraux $\gg^{11}$ ont également accéléré le processus de légalisation nationale et internationale en constituant des « plateformes organisationnelles $\gg^{12}$ exclusivement consacrées à la défense de cette cause. Motivées à l'origine par des préoccupations avant tout morales et sociales, ces ONG œuvrent aujourd'hui à la protection des victimes de la traite. Au-delà du rôle de « filet de sécurité ${ }^{13}$ qu'on leur prête souvent, leur capacité d'immersion fait généralement de ces acteurs sociétaux les seuls interlocuteurs possibles des victimes de la traite, et leur rôle de catalyseur de l'action anti-traite est désormais reconnu et soutenu par la plupart des organisations européennes et internationales ${ }^{14}$. Très vite, cependant, le jeu des influences interétatiques est venu se greffer sur l'action des acteurs privés dans l'émergence d'une politique publique de

8. Les textes précédents ne mentionnaient que les femmes majeures abusées et les enfants. Voir Anne Cazals, Prostitution et proxénétisme en Europe, Paris, La Documentation française, 1995, p. 14-15.

9. Entré en vigueur le 25 décembre 2003.

10. Martha Finnemore, Kathryn Sikkink, «International Norm Dynamics and Political Change », International Organization, 52 (4), 1998, p. 887-917.

11. Ethan Nadelmann, « Global Prohibition Regimes: The Evolution of Norms in International Society », International Organization, 44 (4), 1990, p. 479-526.

12. M. Finnemore, K. Sikkink, «International Norm Dynamics and Political Change », art. cité, p. 887-917.

13. Marie-Claude Smouts, Les organisations internationales, Paris, Armand Colin, 1995, p. 34.

14. Cf. Conseil de l'Europe, La lutte contre la traite des êtres humains aux fins d'exploitation sexuelle: le rôle des ONG, Actes du séminaire, Strasbourg, 29-30 juin 1998. 
lutte contre la traite. Dès 1990, Ethan Nadelmann classe la lutte contre la traite des femmes comme relevant d'un « régime prohibitif global » (global probibition regime) ${ }^{15}$, et donc de l'édiction de normes internationales contraignantes. Les récents instruments adoptés témoignent d'ailleurs d'une volonté de légaliser l'action anti-traite au niveau international et de l'émergence d'une politique publique de lutte contre la traite au niveau européen. La cadence à laquelle sont adoptées les résolutions visant à instaurer un dispositif législatif régional en la matière s'est en effet sensiblement accélérée depuis le milieu des années $1990^{16}$, alimentant «la constitution d'un cadre cognitif commun à un grand nombre d'acteurs publics et privés $\gg^{17}$.

La légalisation précoce et relativement bien acceptée de la lutte internationale contre la traite s'explique non seulement par sa visée universaliste, puisqu'elle édicte des règles s'appliquant à tous et en tous lieux, mais aussi par les références plus ou moins explicites qu'elle contient à d'autres normes unanimement reconnues, telles que la défense de la dignité humaine ou la protection des groupes vulnérables ${ }^{18}$, et, de manière plus ciblée, l'abolition de l'esclavage ou la condamnation des violences faites aux femmes. Non seulement le consensus généralement établi autour de la nécessité pour les États d'unir leurs efforts afin de lutter contre la traite, mais aussi un contexte général de craintes face à l'éventualité d'une poussée migratoire est-européenne et l'interpénétration des concepts de sécurité intérieure et extérieure sont donc à l'origine de la récente multiplication des normes internationales. Le Conseil de l'Europe fait office de pionnier, avec l'adoption dès septembre 1991 d'une recommandation très complète sur l'exploitation sexuelle, la pornographie et le trafic d'enfants et de jeunes adultes. Mais ses initiatives demeurent de portée limitée puisque la « recommandation » en question n'est nullement contraignante pour les États membres. Il en est de même pour la plupart des textes adoptés par l'Union européenne au cours des années 1990, qu'il s'agisse des résolutions du Parlement européen de 1989 et 1993, des recommandations du Conseil de Justice et des Affaires intérieures de novembre 1993, des communications de la Commission européenne de 1996 et 1998 ou des Actions communes adoptées par le Conseil de l'UE en novembre 1996 et février 1997. Toutefois, après l'adoption du Protocole de

15. E. Nadelmann, «Global Prohibition Regimes: The Evolution of Norms in International Society », art. cité, p. $479-526$.

16. Voir Anne Weyembergh, «L'Union européenne et la lutte contre la traite des êtres humains », Cabiers de droit européen, 1-2, 2000, p. 215-251.

17. Yves Surel, «L'intégration européenne vue par l'approche cognitive et normative des politiques publiques », Revue française de science politique, 50 (2), 2000, p. 235-254.

18. M. Finnemore, K. Sikkink, «International Norm Dynamics and Political Change », art. cité, p. 887-917. 
Palerme, le Conseil de l'UE a lui aussi cherché à institutionnaliser la lutte contre la traite et la protection des victimes. Premier instrument européen contraignant, la Décision cadre de 2002, tout en faisant étroitement référence au Protocole de Palerme dans la définition de la traite qu'elle propose ${ }^{19}$, se distingue par sa volonté affichée de prendre davantage en compte la situation spécifique de la victime, et non plus uniquement les agissements des trafiquants.

Pourtant, au niveau international, le débat se caractérise par l'existence d'une multiplicité d'intérêts hétérogènes constitués en advocacy coalitions, ou « coalitions de défense d'une cause $\gg^{20}$. Au cours des négociations sur le Protocole de Palerme, on a vu ainsi s'opposer les acteurs étatiques et les institutions internationales : les premiers craignant que certaines dispositions ne constituent une remise en cause de leur souveraineté (notamment du fait de l'obligation qui leur est faite d'adapter leur législation) ou une charge budgétaire supplémentaire (induite par l'assistance aux victimes ou l'organisation de leur rapatriement), les secondes, partisanes d'un processus de «"sociation" fondé sur la volonté réfléchie, le calcul rationnel et l'acceptation du compromis » ${ }^{21}$, exigeant une approche globale de la part de tous les pays, qu'ils soient d'origine, de transit ou de destination. Au-delà de ce clivage somme toute classique dans les relations internationales, les négociations qui ont précédé l'adoption du Protocole de Palerme ont cependant révélé bien d'autres dissensions: tout d'abord, entre partisans d'un régime prostitutionnel réglementariste, qui considèrent l'exploitation sexuelle comme marginale dans l'industrie du sexe et demandent donc que la traite ne soit réprimée qu'en cas de non-consentement initial de la victime, et défenseurs d'une approche prohibitionniste et/ou abolitionniste, qui écartent le consentement de la victime des éléments à prendre en compte dans les cas de traite ${ }^{22}$; ensuite, entre défenseurs de la liberté du client à acheter des services sexuels, en vertu d'un accord marchand classique, et artisans de sa criminalisation (la Suède en tête $)^{23}$; enfin, entre pays d'origine de la traite, qui demandent pour la plupart que soit conféré un caractère obligatoire aux mesures d'assistance

19. Sont considérés comme constitutifs de la traite le recrutement, le transport, l'hébergement ou la réception de personnes ; le recours à la menace, à la force ou à d'autres formes de coercition, de tromperie et d'abus de pouvoir ; l'objectif final d'exploitation (la prostitution d'autrui ou autres formes d'exploitation).

20. Paul A. Sabatier, « The Advocacy Coalition Framework: Revisions and Relevance for Europe », Fournal of European Public Policy, 5 (1), 1998, p. 98-130.

21. M.-C. Smouts, Les organisations internationales, op. cit., p. 20.

22. C'est finalement cette dernière approche qui a prévalu lors des négociations du Protocole de Palerme, dont l'article 3 stipule que le consentement d'une victime de la traite à l'exploitation envisagée est indifférent.

23. Ce débat semble gagner en vigueur ces derniers temps, notamment dans les pays voisins de la Suède (pays baltes par exemple), et pourrait donc à l'avenir peser davantage sur le débat international. 
et de protection des victimes, et pays de destination, concernés au premier chef par ces dispositions, mais qui ont finalement réussi à faire pencher la balance en faveur de l'optionalité de l'aide aux victimes ${ }^{24}$.

Le Protocole de Palerme n'en est pas moins perçu comme une avancée remarquable, en raison de son rôle tant dans la reconnaissance des droits de la victime que dans l'adoption d'une définition internationalement reconnue et censée mettre fin aux controverses définitionnelles qui avaient jusqu'alors paralysé l'action publique. Il traduit en outre une réelle volonté d'engagement de la part des États parties. Après avoir laissé un temps aux organisations privées le soin de lutter contre le commerce des êtres humains, considéré comme un dysfonctionnement d'ordre moral et humanitaire plus que politique, ceux-ci semblent en effet avoir pris conscience du danger potentiel que représentait, pour leur légitimité nationale, cet énoncé violent de « la possibilité de l'imprévu et de la mort ${ }^{25}$. Dans un contexte politique voué tout entier à la lutte contre la «menace» et à l'«éradication du risque $»^{26}$, la traite n'est plus seulement perçue par les États comme une atteinte à l'individu, appelant une réponse humanitaire, mais aussi comme une des formes d'expression du crime organisé et de l'immigration clandestine, et donc comme une atteinte à l'intégrité du territoire.

C'est justement le télescopage de ces deux types d'approche, humanitaire, d'une part, sécuritaire, d'autre part, mais aussi - et surtout - leur renforcement mutuel, qui semble avoir largement favorisé l'apparition de la lutte contre la traite des femmes sur l'agenda politique européen et international. S'ils semblent à première vue être en compétition sur la scène politique, les discours véhiculés par ces deux coalitions entretiennent de fait une étroite relation d'interdépendance : non seulement l'engagement des gouvernements dans la lutte anti-traite résulte souvent de pressions non gouvernementales, mais la plupart des ONG ne doivent leur existence qu'à l'investissement des gouvernements dans la cause qu'elles défendent, les acteurs étatiques en retirant d'ailleurs eux-mêmes un gain de légitimité ${ }^{27}$. Le fait que la lutte contre la traite ait été constituée en enjeu politique dans les Républiques baltes fraîchement indépendantes illustre bien cet effet de résonance entre les actions gouvernementales et non gouvernementales. Dans ces pays, les premières

24. $C f$. l'article 3 du Protocole de Palerme.

25. Pierre Lascoumes, « Contruction sociale des risques et contrôle du vivant », Revue Prévenir, 24, 1993, p. 23-36.

26. Ibid.

27. C'est le cas du gouvernement lituanien depuis que l'adoption d'un Plan national d'action favorisant la coopération avec le secteur non gouvernemental a permis à la Lituanie d'être classée par le Département d'État américain parmi les pays déployant les plus grands efforts dans la lutte contre la traite. Voir U.S. Department of State, Trafficking in Persons Report 2003: Lithuania, novembre 2003 (http://www.state.gov/g/tip/rls/tiprpt/2003/21276.htm\#lithuania) (consulté le 20.02.2006). 
ONG dédiées à la prévention de la traite ont presque toujours été créées grâce aux efforts des gouvernements occidentaux (nordiques notamment) pour développer la démocratie et favoriser l'émergence d'une société civile, censée faire contrepoids aux autorités administratives et politiques héritées du communisme. Ces acteurs sociétaux « d'importation » ont alors fait pression sur leurs gouvernements respectifs afin que ceux-ci développent une action nationale concertée de lutte contre la traite. Ils sont par exemple à l'origine de l'adoption par le gouvernement lituanien d'un Programme national pour prévenir et contrôler la prostitution et la traite des êtres humains (2002-2004). Au regard des stratégies conflictuelles initialement mises en œuvre par les acteurs sociétaux et gouvernementaux lituaniens, la désignation d'un coordinateur national « neutre » a permis de décloisonner les relations et d'allouer, pour la première fois dans les pays baltes, des moyens étatiques, humains et financiers, à la lutte gouvernementale et non gouvernementale contre la traite ${ }^{28}$.

Signalons également l'impulsion déterminante donnée à l'inscription de la traite sur les agendas politiques par l'Organisation internationale pour les migrations, qui fait de plus en plus office, depuis le milieu des années 1990, de courroie de transmission entre les acteurs nationaux et internationaux. Son positionnement sur plusieurs fronts (migrations clandestines, traite, criminalisation des trafiquants) et sa reconnaissance internationale d'expert des questions migratoires (liée notamment au quasi-monopole qu'elle détient sur la production et la compilation de statistiques relatives à la traite et aux flux migratoires illégaux), auxquels s'ajoute son statut d'organisation internationale à implantation régionale, font en effet de l'OIM un médiateur privilégié entre les acteurs internationaux et locaux de la lutte, mais aussi entre les gouvernements et les ONG. De plus en plus souvent rejointe dans cette démarche médiatrice par des organisations européennes et internationales (OSCE et Conseil de l'Europe notamment, mais aussi, dans une moindre mesure, ONU et UE), l'OIM fait figure de pionnier dans le développement d'une coopération à plusieurs niveaux, dont tous s'accordent à penser qu'elle est indispensable à la mise en place d'une politique globale de lutte contre la traite et de protection des victimes.

Reste que les représentants de chacune des deux principales familles discursives, humanitaire et sécuritaire, continuent majoritairement à se définir par opposition réciproque, quand bien même leur coopération se révèle être un facteur de croissance mutuelle. Il convient donc, sans céder pour autant à une

28. Voir les entretiens conduits par l'auteur avec les acteurs gouvernementaux et non gouvernementaux de la lutte contre la traite en Lituanie en mai 2004. 
vision trop schématique de la dichotomie entre acteurs gouvernementaux et non gouvernementaux, de revenir sur ces deux types de discours - celui qui envisage prioritairement la traite des êtres humains comme un problème migratoire et/ou prostitutionnel, donc de sécurité nationale, d'une part, celui qui défend une conception élargie de la traite comme menace universelle pour le bien de l'humanité et des individus qui la composent, d'autre part-, et d'analyser leur articulation dans l'espace de l'action publique internationale et européenne, ainsi que le rôle de médiateur (policy broker ${ }^{29}$ ) que les institutions internationales et, de plus en plus, européennes sont progressivement appelées à jouer face à ces stratégies conflictuelles.

\section{La bipolarisation des discours}

C'est d'abord comme une menace pour leur sécurité nationale et leur stabilité sociale que les États semblent envisager l'ensemble des migrations illégales, parmi lesquelles ils classent désormais la traite des êtres humains. Ils se trouvent d'ailleurs confortés dans cette approche par la peur que l'immigration suscite en général chez les Européens ${ }^{30}$. La « traite des Blanches », la toutepuissance des «mafias $»^{31}$, la menace d'une « grande invasion $»^{32}$ réveillent des craintes ancestrales et constituent des thèmes facilement exploitables ${ }^{33}$ par les gouvernements pour motiver un renforcement des contrôles aux frontières, premières garantes de l'intégrité territoriale et de l'identité nationale. Faut-il voir dans ces nouvelles menaces des constructions, conscientes ou non, des États pour satisfaire des objectifs économiques et/ou politiques? Force est de reconnaître que, tant que cette spéculation hyperbolique sur les dangers planétaires du crime organisé mobilise l'attention des citoyens, ceuxci sont moins enclins à considérer les éventuels travers (corruption institutionnelle, mauvais résultats économiques) qui conduiraient à une remise en cause de l'action de l'État ${ }^{34}$. Si l'on considère par ailleurs que la menace est souvent une pièce maîtresse dans le processus de construction d'une figure de

29. P. A. Sabatier, «The Advocacy Coalition Framework: Revisions and Relevance for Europe », art. cité, p. 98130.

$30.33 \%$ des Européens se sentent « menacés par l'immigration ». Voir les sondages réalisés par la Fondation NordEst et cités notamment dans Ilvo Diamanti, «Un nouveau mur. L'opinion publique et les immigrés de l'autre rive », Critique internationale, 18, 2003, p. 159-168.

31. Fréquemment évoquée par l'ensemble des acteurs de la lutte anti-traite, gouvernementaux ou non, interrogés en 2004 dans les pays baltes.

32. Didier Bigo, « Comment douter de la sécurité ? », Hommes et Migrations, 1241, 2003, p. 32-42.

33. Margaret E. Beare, «Introduction », dans M. E. Beare (ed.), Critical Reflections on Transnational Organized Crime, Money Laundering and Corruption, Toronto (Canada), University of Toronto Press, 2003.

34. Michael Woodiwiss, «Transnational Organized Crime: The Strange Career of an American Concept », ibid., p. 3-34. 
l'ennemi, dont l'invocation suffit ensuite à expliquer les manques ou les échecs politiques ${ }^{35}$, le concept, même imprécis, galvaudé et largement mythologisé, de «crime transnational organisé » semble pouvoir constituer une ressource pour le politique, dont il contribue à asseoir la légitimité ou du moins à déstabiliser les adversaires ${ }^{36}$. Ainsi l'élargissement à l'Est et le lot d'incertitudes dont il s'accompagne, du fait de la complexification croissante du jeu économique et politique transnationalisé, favorisent-ils la résurgence de rhétoriques sensationnalistes agitant le spectre de la mainmise du crime organisé sur les flux migratoires en provenance de l'Europe du Sud et de l'Est ${ }^{37}$.

Car la menace semble être plus l'exportation du crime des pays en transition vers les pays développés qu'une véritable internationalisation des activités criminelles. À l'origine de ce type de représentation, on trouve notamment la crainte, au sein des organisations policières ouest-européennes, de l'infiltration des polices postcommunistes par le crime organisé. Pareille hantise entretient des discours à résonance « transitologique » dans les polices occidentales qui, bien que promptes à donner des conseils et à prôner la coopération, rechignent encore à instaurer une relation de confiance réciproque, posant ainsi le «modèle occidental » comme but ultime à atteindre par les nouveaux États membres en marche vers la démocratie et l'État de droit ${ }^{38}$. À l'échelle européenne, la conséquence logique de cette conception bipolaire de la criminalité transnationale dans les imaginaires ouest-européens est la partition de l'Europe entre l'Ouest sain et l'Est gangrené ${ }^{39}$, laquelle justifierait la construction d'une « forteresse européenne » et contribue naturellement à attiser la peur de l'étranger chez les citoyens occidentaux.

La corrélation ainsi établie entre crime et migration, et donc entre lutte contre la criminalité et fermeture des frontières, conduit progressivement à un « effacement discursif, organisationnel et instrumental ${ }^{40}$ de la distinction entre traite, trafic, migration illégale et crime organisé. Ce brouillage des catégories est d'ailleurs entretenu, au niveau européen, par la définition du

35. Didier Bigo, « Du discours sur la menace et ses ambiguïtés », Les Cahiers de la Sécurité intérieure, 14, 1993, p. 179196.

36. Voir notamment Gilles Favarel-Garrigues, « Concurrence et confusion des discours sur le crime organisé en Russie », Cultures et Conflits, 2001, 42, p. 9-46.

37. Confirmé par des entretiens conduits en République tchèque et en Autriche en 2005.

38. Didier Bigo, « La coopération policière avec les PECO : entre confiance et exigence », Les Cabiers de la Sécurité intérieure, 41, 2000, p. 141-174. Voir aussi les entretiens conduits par l'auteur avec la police et des représentants de la classe politique estonienne.

39. James Sheptycki, «Against Transnational Organized Crime», dans M. E. Beare (ed.), Critical Reflections on Transnational Organized Crime, Money Laundering and Corruption, op. cit., p. 120-144.

40. Monica den Boer, « Crime et immigration dans l'Union européenne », Cultures et Conflits, 31-32, 1998, p. 101124. 
mandat d'Europol, qui identifie la lutte contre les migrations illégales comme un domaine d'action prioritaire de la lutte contre la criminalité au même titre que le terrorisme, le trafic de drogue ou la traite des êtres humains, et, sur la scène internationale, par le choix des Nations unies d'inscrire le Protocole de Palerme dans la Convention contre la criminalité transnationale organisée. À l'échelle nationale, le flou entourant les notions de crime, traite, trafic et migration illégale se traduit, entre autres, par leur prise en charge globale par un seul et même département de police, ainsi que par l'amalgame couramment pratiqué dans la collecte de données chiffrées. Il en résulte une focalisation systématique sur la lutte contre les groupes criminels et les migrations clandestines. Dès lors, les femmes ayant fait l'objet d'opérations de traite sont avant tout considérées comme des «sujets de droit pénal », dont la protection n'est pas prioritairement justifiée par des considérations humanitaires mais par la nécessité de faciliter l'action de la police et de la justice. Les avantages que les victimes pourraient trouver à accepter de témoigner sont alors assez minces : non seulement le permis de séjour qu'on leur promet à titre de récompense n'est en général que temporaire, l'objectif étant qu'elles soient présentes uniquement pendant la durée du procès, mais elles ne tirent aucun bénéfice des confiscations des biens des trafiquants auxquelles les gouvernements sont tenus de procéder sur injonction des Nations unies ${ }^{41}$. Par ailleurs, de nombreuses condamnations sont toujours prononcées à leur encontre pour migration illégale ou travail clandestin, sans reconnaissance de leur statut de victime ${ }^{42}$.

Le ciment de cet amalgame entre crime et migration est à la fois la croyance largement partagée dans le caractère nécessairement « mafieux » de ces activités et le langage utilisé pour l'argumentation. Indéniablement doté d'un « pouvoir linguistique de constitution ${ }^{43}$ et donc de construction de la subjectivité, ce langage s'articule autour d'une rhétorique moralisatrice, catastrophiste, et surtout guerrière (on parle de «lutte» au nom de la «sécurité nationale $\gg$ contre de $\ll$ nouvelles menaces $\gg$ ), qui induit des réponses d'ordre exclusivement militaire et policier. Celles-ci ne rendent pourtant compte que

\footnotetext{
41. Les participants aux négociations sur la Convention des Nations unies contre la criminalité transnationale organisée ont refusé que les biens confisqués servent à financer les programmes d'aide aux victimes et de prévention de la traite. Voir Ann D. Jordan, « Human Rights or Wrongs ? The Struggle for a Rights-based Response to Trafficking in Human Beings », Gender and Development, 10 (1), 2002, p. 28-37.

42. Selon l'Office fédéral de police criminelle allemand (Bundeskriminalamt), $24 \%$ des victimes de la traite avaient été expulsées en 2001, 13 \% seulement étaient retournées volontairement dans leur pays d'origine et $13 \%$ également avaient pu bénéficier d'un permis de séjour temporaire. Voir Organisation internationale pour les migrations, «La traite en Allemagne», Traite des migrants (Bulletin trimestriel), 27, 2003, p. 2 (http://www.iom.int//DOCUMENTS/PUBLICATION/FR/tm27_fr. pdf) (consulté le 20.02.2006). Le Protocole de Palerme, malgré l'avancée qu'il représente indéniablement dans la prise en considération des droits de la victime, ne garantit pas qu'il ne soit procédé à aucune expulsion contre la volonté de la victime.

43. Judith Butler, Le pouvoir des mots : politique du performatif, Paris, Éditions Amsterdam, 2004, p. 64.
} 
d'une certaine construction des faits et montrent donc rapidement des signes d'essoufflement face à une réalité plus complexe. Elles reposent tout d'abord sur une distinction par trop simpliste entre migration légale et migration illégale. En théorie, la traite constituerait un sous-ensemble de l'immigration illégale. Dans la pratique, les victimes de la traite pénètrent souvent en toute légalité sur le territoire d'un État, et la tromperie et l'exploitation sont loin d'être l'apanage des schémas de migration de main-d'œuvre illégale ${ }^{44}$. Par ailleurs, la partition implicite du monde entre Bons et Mauvais, d'où semble découler l'argumentation sécuritaire, occulte un aspect important du développement de ces nouvelles migrations, à savoir la demande occidentale. Il faut pourtant convenir que les migrants illégaux employés dans l'économie souterraine, industrie du sexe comprise, satisfont une demande spécifique dans les pays économiquement avancés ${ }^{45}$. Ainsi la prévalence de préjugés racistes et culturalistes dans l'industrie du sexe en Occident explique-t-elle l'engouement des clients pour les prostituées d'origine étrangère (et notamment d'Europe de l'Est), traditionnellement considérées non seulement comme exotiques et donc plus désirables ${ }^{46}$, mais aussi comme moins émancipées et donc plus « fiables », car moins susceptibles de s'opposer à la volonté de leur partenaire ${ }^{47}$.

Or, à partir du moment où une demande existe, aucun régime migratoire, aussi répressif soit-il, ne peut endiguer le flux des personnes susceptibles de répondre à cette demande. Confirmant l'énoncé de Foucault selon lequel «l'existence d'un interdit légal crée autour de lui un champ de pratiques illégalistes $»^{48}$, le renforcement des contrôles aux frontières, loin de réduire le champ d'activité du crime organisé, provoque une augmentation continuelle des trafics ${ }^{49}$, dont le corollaire est l'accroissement du phénomène de vulnérabilisation des migrants.

44. Bridget Anderson, Julia O'Connell Davidson, Is Trafficking in Human Beings Demand Driven? A Multi-Country Pilot Study, Genève, IOM Migration Research Series, 2003, p. 10.

45. Vincenzo Ruggiero, «Trafficking, Immigration and Invisibility », Document présenté à la conférence New Frontiers of Crime: Trafficking in Human Beings and New Forms of Slavery organisée par les Nations unies à Vérone les 22 et 23 octobre 1999.

46. Voir notamment Laura Keeler, Marjut Jyrkinen, « Racism in the Sex Trade in Finland », NIKK magasin (Nordic Institute for Women's Studies and Gender Research), 1, 2002, p. 33-36.

47. Voir Riitta Vartti, «German Matchmaking Websites: Online Trafficking in Women ? », Sexuality and Culture, 5 (3), 2001, p. 49-76. Voir aussi les entretiens conduits par l'auteur avec les clients germanophones de la prostitution en République tchèque (2005).

48. Michel Foucault, Surveiller et punir, Paris, Gallimard, 1975, p. 285.

49. Europol estime ainsi que, sur les 500000 personnes entrant chaque année dans les États membres, la moitié est aidée par des trafiquants et des groupes criminels. Voir Willy Bruggeman, « Illegal Immigration and Trafficking in Human Beings Seen as a Security Problem for Europe », European Conference on Preventing and Combating Trafficking in Human Beings: Global Challenge for the 21st Century, Bruxelles, 18-20 septembre 2002. Voir également Andreas Schloenhardt, « Organized Crime and the Business of Migrant Trafficking: An Economic Analysis », Crime, Law and Social Change, 32 (3), 1999, p. 203-233. 
De même, les politiques de lutte contre la traite qui s'appuient sur une approche répressive de la prostitution induisent, elles aussi, des effets pervers incontrôlés. En Suède, l'adoption en 1999 d'une loi visant à criminaliser les clients de la prostitution n'a pas empêché l'augmentation du nombre de femmes victimes de la traite ${ }^{50}$. De fait, les mesures prohibitionnistes confèrent encore plus de pouvoir aux réseaux criminels, les proxénètes se voyant dès lors investis d'un rôle de «protecteur » aux yeux des femmes qui travaillent pour eux parce qu'ils négocient directement avec le client ${ }^{51}$ et se chargent d'organiser sa rencontre clandestine avec la prostituée. De telles politiques sont en outre à l'origine de véritables « croisades morales ${ }^{52}$ contre les prostituées, croisades encore plus virulentes si les prostituées sont étrangères. On a vu ainsi ressurgir dans certains pays d'Europe de l'Est une rhétorique purificatrice prônant l'éradication de l'«attitude parasitaire ${ }^{53}$ des prostituées, qui n'est pas sans rappeler la rhétorique socialiste de lutte contre le parasitisme ${ }^{54} \ldots$ Les résultats des enquêtes conduites par l'OIM dans le cadre de la campagne d'information contre la traite des femmes organisée en 2002 dans les pays baltes confirment la propension des populations à rejeter la faute sur les victimes accusées d'êtres immorales, crédules ou tout simplement idiotes $^{55}$. 53,4 \% des personnes interrogées en Lituanie estiment ainsi que ce sont les victimes elles-mêmes qui, pour avoir accordé trop rapidement leur confiance, portent la plus grande part de responsabilité dans le développement de la traite à des fins d'exploitation sexuelle ${ }^{56}$. Par ailleurs, à peine plus de $20 \%$ des Estoniens et des Lituaniens se prononcent en faveur de l'assistance aux victimes ayant choisi d'émigrer en sachant qu'elles devraient travailler comme prostituées, et donc considérées comme initialement « consentantes ${ }^{57}$.

En effet, la conception de la victime qui prévaut encore aujourd'hui est, comme au début du siècle dernier ${ }^{58}$, celle de la « madone $»$, la jeune fille innocente,

50. A. D. Jordan, « Human Rights or Wrongs ? The Struggle for a Rights-based Response to Trafficking in Human Beings », art. cité, p. 28-37.

51. Passible de six mois d'emprisonnement.

52. Richard H. Friman, « Prise au piège de la folie ? Le pouvoir étatique et le crime organisé transnational dans l'œuvre de Susan Strange », Cultures et Conflits, 42, 2001, p. 139-160.

53. Marion Pajumets, Prostitution: A Social Problem? The Views on Prostitution's Nature, Causes and Effects in the Baltic States and North-Western Russia, Tallinn, Estonian Women's Studies and Resource Centre, 2004, p. 51 (http:// www.childcentre.info/news/estonia/dbaFile11288.pdf) (consulté le 20.02.2006).

54. Dominique Colas, Le léninisme, Paris, PUF, 1998, p. 201-207.

55. M. Pajumets, Prostitution: A Social Problem? The Views on Prostitution's Nature, Causes and Effects in the Baltic States and North-Western Russia, op. cit., p. 51.

56. Igoris Bazylevas, Renaldas Žekonis, Prevention and Control of Trafficking in Human Beings in Lithuania, Vilnius, S. Smirnovo II, 2003, p. 23.

57. OIM, Public Perception and Awareness of Trafficking in Women in the Baltic States (2), Vilnius, OIM, 2002, p. 24.

58. A. Corbin, Les filles de noce. Misère sexuelle et prostitution aux 19et $20^{e}$ siècles, op. cit., p. 427. 
contrainte et dupée, tandis que la victime consentante, bien que tout autant exploitée, reçoit l'étiquette de «mauvaise fille». De la catégorisation de la victime dépend souvent l'assistance dont elle bénéficie ensuite, la plupart des acteurs (gouvernementaux ou non) se montrant plus enclins à aider les «madones ${ }^{59}$. Cette tendance à la catégorisation simplificatrice, d'ailleurs fréquemment relayée par les médias, encourage les analyses polarisées et conduit à voir dans la « madone » la seule vraie victime possible. Cette mauvaise interprétation du témoignage de la victime initialement « consentante » en une confession de complicité n'est pas sans rappeler l'analyse des discours sexualisés fournie par Judith Butler ${ }^{60}$. La parole de la victime de la traite qui reconnaît avoir été sexuellement disponible, de par son assimilation à une « puissance d'agir » sexuelle, est considérée comme un aveu de culpabilité et conforte l'institution d'une discrimination entre victimes «innocentes », méritant une protection, et victimes « coupables », expulsées sur une décision unilatérale par les services de police.

La politique européenne d'assistance aux victimes est elle-même accusée d'institutionnaliser une forme de discrimination entre victimes méritantes et non méritantes. S'alignant sur la position de la plupart des institutions internationales et de certains États membres (Allemagne, Belgique et Pays-Bas notamment), et faisant fi des affirmations selon lesquelles la traque des trafiquants « par le bas » (c'est-à-dire par le biais des victimes) serait moins efficace que « par le haut » (par le contrôle des flux financiers notamment) ${ }^{61}$, la Décision cadre de 2002 subordonne l'octroi d'un permis de séjour aux victimes de la traite à la volonté de celles-ci de témoigner contre leurs trafiquants. Cette orientation paraît pourtant moralement discutable : en marchandant ainsi leur protection, les États entretiennent une démarche sur-responsabilisante parfois qualifiée $\mathrm{d}^{\prime} \ll$ abus de vulnérabilité » à l'égard des victimes, condamnées à choisir entre témoigner, et donc s'exposer aux représailles des trafiquants, ou être rapatriées pour séjour illégal et/ou exercice de la prostitution, et courir ainsi le risque d'être revictimisées ${ }^{62}$.

Face au discours sécuritaire axé sur un double contrôle migratoire et prostitutionnel, les organisations non gouvernementales et, de plus en plus, les organisations internationales défendent une position « humanitaire » au sens premier du terme, c'est-à-dire qui a « pour objet le bien universel de l'huma-

\footnotetext{
59. Hypothèses confirmées par les entretiens menés au printemps dernier avec différents acteurs de la lutte contre la traite (ONG, représentants de la police criminelle et officiers gouvernementaux notamment) dans les pays baltes.

60. J. Butler, Le pouvoir des mots : politique du performatif, op. cit., p. 137.

61. Affirmations confirmées par les représentants de la police criminelle estonienne interrogés au printemps 2004.

62. C'est-à-dire d'être à nouveau victime des trafiquants, une fois rapatriée.
} 
nité $\gg$, et énoncent le primat du social sur le politique ${ }^{63}$. Animés par un idéal de justice universelle et de défense des valeurs telles que l'empathie, l'altruisme ou l'engagement idéationnel ${ }^{64}$, les tenants de cette approche appellent à considérer avant tout la traite comme une violation des droits humains fondamentaux. Ils prônent donc la reconnaissance du statut de victimes, et non de criminelles, des femmes ayant fait l'objet d'une opération de traite et dénoncent l'absence de systématisation des mesures d'assistance. Outre la tendance des services de police à n'accorder le statut de victime qu'aux femmes initialement non consentantes à l'émigration prostitutionnelle (et ce malgré l'adoption du Protocole de Palerme déclarant le consentement comme un facteur indifférent), les relations entre acteurs institutionnels et non institutionnels de la lutte contre la traite (police et ONG notamment) sont encore souvent teintées de méfiance et d'hostilité réciproques ${ }^{65}$. Seule une intensification de la coopération à la fois locale, nationale et internationale permettrait pourtant de réduire le poids des facteurs aléatoires, humains ou financiers, dans le déclenchement d'une procédure d'assistance aux victimes de la traite et de diminuer ainsi le nombre de femmes expulsées et non assistées à leur retour, pour lesquelles le risque de persécution, voire de revictimisation par les trafiquants est particulièrement élevé. Sans compter que leurs droits à la protection de la vie privée sont fréquemment bafoués et les motifs de leur expulsion (activité prostitutionnelle et/ou séjour illégal) divulgués dans leur communauté d'origine, ce qui les expose à la stigmatisation et à la discrimination, voire à des tentatives de chantage de la part des autorités locales ${ }^{66}$. Pour que l'aide aux victimes soit institutionnalisée, les partisans de l'approche humanitaire réclament donc l'adoption de lois dures, précises et contraignantes, au nom d'un sens moral universel ${ }^{67}$.

Il arrive pourtant que dans ce combat pour la défense des droits fondamentaux et le bien de l'humanité « la sensiblerie (...) fini[sse] par submerger l'internationalisme $»^{68}$. Cédant à la tentation du profilage normatif ${ }^{69}$, les « humanitaires » ont tendance à porter sur les victimes de la traite un regard fortement teinté de misérabilisme. Ils ne voient en elles que de jeunes

63. Maurice Tournier, « Humanitaire est-il apolitique de naissance ?», Mots, 65, 2001, p. 136-145.

64. M. Finnemore, K. Sikkink, «International Norm Dynamics and Political Change », art. cité, p. 887-917.

65. Vérifié lors des entretiens conduits en avril 2004 avec des représentants d'ONG et de la police criminelle estonienne.

66. OIM, « Nouvelles études de l'OIM sur la traite », Traite des migrants (Bulletin trimestriel), 24, 2001, p. 4 (http:/ /www.iom.int//DOCUMENTS/PUBLICATION/FR/tm24_FR. pdf) (consulté le 20/02/2006).

67. E. Nadelmann, «Global Prohibition Regimes: The Evolution of Norms in International Society », art. cité, p. $479-526$.

68. M. Tournier, «Humanitaire est-il apolitique de naissance ? », art. cité, p. 136-145.

69. Voir Erving Goffman, Stigmate. Les usages sociaux des handicaps, Paris, Les Éditions de Minuit, 1975, p. 68. 
femmes innocentes poussées par la misère à subir les exigences des trafiquants, des « victimes impuissantes d'un système tout puissant ${ }^{70}$, passives, vulnérables, voire incapables de jugements raisonnés ${ }^{71}$. Certaines campagnes de prévention de la traite des femmes illustrent bien la prévalence de ces perceptions résolument occidentales des victimes, présentées comme écrasées par le poids des traditions et donc survictimisées ${ }^{72}$, tandis que les femmes occidentales se positionnent au contraire en sauveuses émancipées et civilisées $^{73}$. Ce type d'approche tend à considérer, à tort, la parole des victimes comme systématiquement caduque ${ }^{74}$. L'activité prostitutionnelle des victimes de la traite conduit souvent les observateurs à ne les considérer qu'à travers des mécanismes sociaux de contrôle, de stigmatisation et de soumission (à l'égard des clients, des proxénètes, des policiers) et à «méconnaître toutes les possibilités de jeu dont elles disposent pour faire face à ces mécanismes de domination $»^{75}$. C'est la raison pour laquelle la plupart des organisations chargées d'assister les victimes rapatriées ne comprennent absolument pas la décision de certaines d'entre elles de repartir «travailler » pour les mêmes trafiquants.

L'analyse des discours humanitaires à vocation universaliste sur la lutte contre la traite semble alors donner raison à Judith Butler, qui affirme qu'on ne peut supposer l'universel préétabli sans courir le risque de l'ethnocentrisme ou de l'« impérialisme culturel ${ }^{76}$.

\section{Le statut de victime de la traite}

C'est finalement dans la codification des exclusions que discours sécuritaires et humanitaires, aussi dissonants qu'ils se veuillent, semblent se rejoindre. Négligeant toute approche sociologique des victimes de la traite à des fins d'exploitation sexuelle, ils laissent souvent dans l'ombre certaines spécificités de leur condition, notamment l'ambiguïté de leurs liens avec leurs trafi-

70. Françoise Guillemaut, Femmes et Migrations. Les femmes venant d'Europe de l'Est, Lyon, Le Dragon Lune - Cabiria Éditions, 2002, p. 14.

71. A. D. Jordan, « Human Rights or Wrongs ? The Struggle for a Rights-based Response to Trafficking in Human Beings », art. cité, p. 28-37.

72. Stéréotype auquel la campagne d'information menée par l'OIM dans les pays baltes en 2002 n'échappe d'ailleurs pas complètement, puisqu'elle présente la victime de la traite sous l'apparence d'une marionnette (ce qui suppose non seulement qu'elle est manipulée, mais aussi que celui qui la manipule demeure invisible).

73. Barbara Sullivan, « Feminism and New International Law », International Feminist fournal of Politics, 5 (1), 2003, p. 67-91.

74. Daniel Welzer-Lang, Odette Barbosa, Lilian Mathieu, Prostitution : les uns, les unes et les autres, Paris, Anne-Marie Métailié, 1994, p. 216.

75. D. Welzer-Lang, O. Barbosa, L. Mathieu, Prostitution : les uns, les unes et les autres, op. cit., p. 216.

76. J. Butler, « Considérer le problème plus que l'identité », Mouvements, 29, 2003, p. 123-131. 
quants. Certes, les formes de solidarité qui caractérisent les relations au sein de la communauté prostitutionnelle sont pour le moins réduites ${ }^{77}$, mais il peut arriver que les victimes éprouvent à l'égard de leurs trafiquants, qui sont la plupart du temps leurs compatriotes ${ }^{78}$, des sentiments pouvant aller jusqu'au refus de les dénoncer. Cette solidarité d'exilés, mêlée de peur et de mystification, peut se transformer en une véritable dépendance affective ${ }^{79}$. Quelle que soit la tournure que prennent les événements, le trafiquant demeure avant tout le « protecteur » dans l'imaginaire de la victime $:$ il est le premier à lui avoir donner l'espoir d'une vie meilleure, et, même si elle n'est finalement pas dupe, la victime s'accroche à ce mensonge, ponctuellement ravivé par quelques belles promesses, et entretient d'elle-même le mythe de l'amour romantique ${ }^{80}$ pour combler un vide relationnel. Rappelons qu'à cette dépendance affective s'ajoute de plus en plus souvent, une dépendance physique due à la toxicomanie. La manipulation, la fragilisation psychologique qu'elle entraîne et le contrôle permanent dont elle s'accompagne rendent presque impossible la moindre tentative d'autonomisation de la victime. $\mathrm{La}$ définition de l'individu stigmatisé, « que quelque chose disqualifie et empêche d'être pleinement accepté par la société », énoncée par Erving Goffman ${ }^{81}$, s'applique tout particulièrement à la victime de la traite exerçant la prostitution. La première manifestation comportementale du stigmate est le dualisme dissimulation/révélation en vertu duquel, tout en se dévoilant aux clients, trafiquants, ou autres membres de la communauté prostitutionnelle, la victime n'aura de cesse de dissimuler ses activités à ses proches (à supposer qu'elle ait pu rester en contact avec eux). Elle se trouve ainsi placée dans une double situation de non-vérité ${ }^{82}$ : par rapport à sa famille, pour laquelle elle s'invente une autre vie, et par rapport à sa propre identité, dont on la force à se détacher (en lui confisquant ses papiers ou en falsifiant son nom et son âge au gré des villes pour brouiller les pistes) ou dont elle cherche à se détacher pour se protéger, comme si pratiquer la prostitution en endossant une autre identité pouvait préserver son intégrité ${ }^{83}$. Cette tentative de distanciation et l'isolement qui en découle engendrent des troubles psychologiques pouvant

\footnotetext{
77. L. Mathieu, «L'espace de la prostitution. Éléments empiriques et perspectives en sociologie de la déviance », Sociétés contemporaines, 38, 2000, p. 99-116. C'est ce que confirment également les entretiens réalisés auprès d'acteurs du milieu prostitutionnel (prostituées notamment) en Lituanie (2004).

78. OIM, La traite des mineurs non accompagnés dans l'Union européenne. Allemagne, Belgique, Espagne, France, Grèce, Italie, Pays-Bas, Paris, Institut des hautes études de la Sécurité intérieure, 2002, p. 90.

79. F. Guillemaut, Femmes et Migrations. Les femmes venant d'Europe de l'Est, op. cit., p. 40.

80. Ibid., p. 55.

81. E. Goffman, Stigmate. Les usages sociaux des handicaps, op. cit., p. 7.

82. F. Guillemaut, Femmes et Migrations. Les femmes venant d'Europe de l'Est, op. cit., p. 47.

83. Ibid., p. 50.
} 
aller jusqu'à l'éclatement de la personnalité ${ }^{84}$, le corps, instrument de l'accumulation économique, devenant un « corps-pour-autrui $»^{85}$.

Constamment tiraillée entre vérité et non-vérité, reconnaissance du stigmate et dissimulation, soumission à la force et mythe romantique, la victime estelle capable de développer une quelconque stratégie d'autonomie ? Négligeant la démarche initialement volontariste de nombre de ces femmes, candidates à l'émigration malgré les dangers potentiels de l'entreprise, sécuritaires et humanitaires s'accordent à nier la capacité de la victime à développer une telle stratégie. Il y a pourtant, de plus en plus souvent (en Europe de l'Est notamment), consentement initial de la part des victimes, avant que la violence et la contrainte ne viennent l'anéantir. En 1995, un rapport de l'OIM a révélé que la plupart des jeunes femmes originaires d'Europe centrale et orientale et victimes de la traite à destination des pays occidentaux savaient quel genre de travail on attendait d'elles, même si elles ignoraient les conditions dans lesquelles il leur faudrait effectuer ce travail parce qu'elles avaient une image faussée - presque positive - de la prostitution en Europe de l'Ouest ${ }^{86}$. Par ailleurs, malgré la domination qu'elles subissent, certaines victimes parviennent à développer non seulement des tactiques de survie mais aussi parfois de réelles stratégies d'autonomie qui leur permettent d'échapper au contrôle des trafiquants, et auxquelles elles doivent donc leur salut. Ainsi les relations entre trafiquants et victimes nous semblent-elles devoir être comprises au sens foucaldien de pouvoir produisant un contre-pouvoir ${ }^{87}$. La reconnaissance du potentiel de résistance et d'autonomie des victimes conditionne en effet le regard ultérieur porté sur elles, et notamment la propension des acteurs à les associer au processus de leur reconstruction psychologique, souvent mené d'autorité, aujourd'hui encore, par des acteurs sociaux convaincus que ces femmes ne se sentent pas vraiment concernées dès qu'il s'agit de réveiller une souffrance qu'elles ont cherché à fuir ${ }^{88}$. Et pourtant, l'on ne dépasse pas une souffrance sans la répéter : « sa répétition est à la fois la continuation du traumatisme et ce qui marque une distance au sein même de la structure du traumatisme ${ }^{89}$. S'il «n'est pas possible de ne pas

\footnotetext{
84. Fondation Scelles, La prostitution adulte en Europe, Toulouse, Éditions Érès, 2002, p. 45.

85. L. Mathieu, «L'espace de la prostitution. Éléments empiriques et perspectives en sociologie de la déviance », art. cité, p. 99-116.

86. OIM, Trafficking and Prostitution: The Growing Exploitation of Migrant Women from Central and Eastern Europe, Budapest, OIM, 1995. Voir aussi Europol, Trafficking in Human Beings for Sexual Exploitation. A European Perspective, La Haye, Europol, 2002, p. 4.

87. M. Foucault, Histoire de la sexualité, tome 1, La volonté de savoir, Paris, Gallimard, 1976, p. 125-127.

88. Amicale du Nid, Assises nationales sur la prostitution : s'associer pour réussir la réinsertion, Paris, UNESCO, 27 mars 1992.

89. J. Butler, Le pouvoir des mots : politique du performatif, op. cit., p. 164.
} 
répéter $\gg^{90}$, reste cependant à savoir comment, où et pour quoi cette confrontation avec le traumatisme des victimes doit avoir lieu.

L'action européenne est-elle alors en mesure de favoriser une meilleure prise en compte de la victime dans les politiques anti-traite? La Conférence de l'Union européenne tenue à Bruxelles du 18 au 20 septembre 2002 sur la prévention et la lutte contre la traite des êtres humains, et la Déclaration de Bruxelles ${ }^{91}$ à laquelle elle a donné lieu, constituent sans doute les prises de position les plus claires de la part des instances européennes en faveur du développement d'une forme de sociologie de la victime. La Déclaration appelle en effet à renforcer les réseaux européens d'assistance aux victimes et à multiplier les études portant sur le profil de ces dernières, afin de mieux comprendre les conditions de leur réintégration et surtout d'éviter leur revictimisation, stigmatisation, criminalisation ou persécution. Certes, il ne s'agit encore que d'une déclaration d'intention ne présentant aucun caractère contraignant pour les États parties, mais elle reflète clairement le souci relativement nouveau des institutions européennes de se poser en médiateur entre les différents acteurs et niveaux engagés dans la lutte, et pourrait éventuellement favoriser l'introduction de changements dans le droit ou la procédure pénale des États membres.

Malgré ces récentes avancées, c'est dans le domaine de la protection des droits des victimes que l'Union est toujours en butte aux critiques les plus virulentes. Son action dans ce domaine est jugée minimale ${ }^{92}$ par le Haut Commissariat des Nations unies pour les droits de l'homme et les réfugiés ${ }^{93}$, qui lui reproche de préférer la répression des groupes criminels organisés à l'assistance aux victimes. Par ailleurs, le caractère encore embryonnaire de la coopération entre les États membres rend difficile l'émergence d'une approche intégrée et la tentation transitologique des organisations (policières notamment) d'Europe de l'Ouest, que nous évoquions plus haut, instaure une coopération de facto inégalitaire car supposant l'adaptation, voire l'obéissance des États anciennement socialistes et candidats à l'UE à un modèle proposé par l'Europe de l'Ouest ${ }^{94}$. Plus généralement, la légalisation européenne de

\footnotetext{
90. Ibid.

91. Commission européenne, « Brussels Declaration on Preventing and Combating Trafficking in Human Beings »,

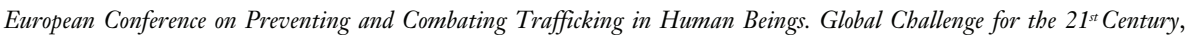
Bruxelles, 18-20 septembre 2002.

92. La Décision cadre ne prévoit aucune assistance pour les victimes après la fin du procès et maintient de surcroit la discrimination évoquée plus haut entre les victimes « méritant» une protection (car disposant d'informations susceptibles de faciliter l'action de la justice) et celles qui n'en sont pas jugées dignes.

93. Tom Obokata, «EU Council Framework Decision on Combating Trafficking in Human Beings: A Critical Appraisal », Common Market Law Review, 40, 2003, p. 917-936.

94. D. Bigo, « La coopération policière avec les PECO : entre confiance et exigence », art. cité.
} 
la lutte contre la traite se heurte toujours, outre aux velléités concurrentielles des différents acteurs engagés dans la lutte, à une définition trop vague et fluctuante de la traite (résultant entre autres de la superposition de différents ensembles de normes européennes) et donc à la difficulté de collecter des données, étant donné l'amalgame couramment pratiqué entre traite, trafic et migrations illégales. Sans compter qu'il n'est même pas certain que l'édiction de normes internationales destinées à lutter contre la traite participe de la réduction effective du phénomène à l'échelle planétaire : outre que les questions prostitutionnelles semblent avoir été, tout au long du $\mathrm{XX}^{\mathrm{e}}$ siècle, particulièrement résistantes aux mesures de justice criminelle ${ }^{95}$, la traite, bien qu'unanimement condamnée, présente certaines caractéristiques qui rendent sa prohibition particulièrement difficile, à savoir non seulement la présence de ressources abondantes et aisément accessibles (en l'occurrence, les femmes), mais aussi l'existence d'une demande occidentale peu ou pas réductible ${ }^{96}$. Il est donc légitime de penser que le déclin éventuel des activités de traite des femmes, à l'échelle européenne comme internationale, procédera d'évolutions de nature sociale, économique ou démographique, et non, en premier lieu, de l'édiction de normes internationales prohibitives.

C'est donc plus à leur «dimension symbolique ${ }^{97}$ qu'à leur efficacité concrète que se mesurent les politiques européennes de lutte contre la traite. Il faut alors leur reconnaître la capacité de créer, par la construction d'une nouvelle représentation du problème et par l'accélération de sa mise sur agenda, les « cadres intellectuels et normatifs qui déterminent les grandes orientations des politiques publiques $\gg$ nationales ${ }^{98}$. À ce titre, elles ont une triple fonction de légitimation : tout d'abord, en cherchant à concilier les intérêts des différents acteurs en présence, elles jouent de plus en plus souvent un rôle de médiateur et légitiment la participation des groupes de défense d'une cause, privés notamment, au processus décisionnel ; ensuite, elles constituent une source internationale de légitimation pour les entrepreneurs de normes domestiques qui, défendant une position minoritaire à l'échelle nationale, invoquent les normes européennes pour renforcer leur position dans le débat interne ${ }^{99}$; enfin, elles sont un des principaux moyens de légitimation tant nationale qu'internationale des États, auxquels elles per-

95. E. Nadelmann, « Global Prohibition Regimes: The Evolution of Norms in International Society », art. cité, p. $479-526$.

96. Ibid.

97. Pierre Muller, Yves Surel, L'analyse des politiques publiques, Paris, Montchrestien, 1998, p. 27.

98. Ibid., p. 99.

99. M. Finnemore, K. Sikkink, «International Norm Dynamics and Political Change », art. cité, p. 887-917. On observe par exemple un recours massif des ONG estoniennes aux normes européennes pour justifier leur action et dénoncer l'absence d'engagement gouvernemental dans le domaine de la lutte contre la traite. 
mettent de renforcer la crédibilité de leurs engagements ${ }^{100}$. Il convient donc de se garder de toute interprétation schématique du processus de légalisation de la lutte contre la traite, en dépit de la bipolarisation discursive dans laquelle le débat européen semble empêtré. Loin de traduire exclusivement la volonté unilatérale des États qui cherchent à satisfaire leur propre intérêt (hypothèse rationaliste pure) ou les talents de persuasion des acteurs non étatiques épris de moralité (hypothèse constructiviste ou normative), la loi est au contraire une «entreprise normative et intéressée ${ }^{101}$. Si l'affect et l'empathie ne sauraient constituer l'unique moteur de l'action internationale dans ce domaine, prétendre qu'ils n'existent pas «serait méconnaître la dynamique fondamentale de la vie politique ${ }^{102}$. Cependant, de même qu'il ne saurait y avoir de politique européenne de lutte contre la traite sans considérations morales, de même toute démarche reposant uniquement sur des facteurs émotionnels ou des sentiments humanitaires, tels que la compassion ou la foi absolue en l'universalisme, court le risque de verser dans l'ethnocentrisme et l'impérialisme culturel ${ }^{103}$.

Mathilde Darley est doctorante de I'IEP de Paris au Centre français de recherche en sciences sociales (CEFRES) de Prague. Elle a participé à l'élaboration de programmes de prévention de la traite des femmes en Estonie en 2002-2003. Ses recherches actuelles portent notamment sur le dispositif de lutte contre les migrations clandestines en République tchèque et en Autriche ainsi que sur la prostitution frontalière en République tchèque. Adresse électronique : mathilde@cefres.cz

100. Kenneth W. Abbott, Duncan Snidal, « Hard and Soft Law in International Governance », International Organization, 54 (3), 2000, p. 421-456.

101. Ibid.

102. M. Finnemore, K. Sikkink, «International Norm Dynamics and Political Change », art. cité, p. 887-917.

103. Ce texte doit beaucoup aux précieux commentaires de Gilles Favarel-Garrigues ainsi qu'à la relecture attentive des correcteurs anonymes. 